\section{Sister Mary Joseph Nodule}

Parth Desai, DO; Naser Yamani, MD

From the Department of Internal Medicine at John

H. Stroger, Jr. Hospital of Cook County in Chicago, Illinois.

Financial Disclosures: None reported.

Support: None reported.

Address correspondence to

Parth Desai, DO, John

H. Stroger, Jr. Hospital of Cook

County, 1969 Ogden Ave,

Chicago, IL 60612-3765.

Email: parth.desai@

cookcountyhhs.org

Submitted

December 19, 2019;

revision received

March 5, 2020;

accepted

March 20, 2020

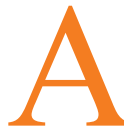

59-year-old woman with ductal carcinoma in situ status post-bilateral mastectomy presented to the emergency department with two weeks of jaundice. Vital signs were normal and examination revealed marked scleral icterus, jaundice, and a palpable subcutaneous umbilical nodule. Laboratory investigation revealed direct hyperbilirubinemia with direct bilirubin of $16.2 \mathrm{mg} / \mathrm{dL}$ (Ref: 0-0.2 mg/dL), total bilirubin of $22.3 \mathrm{mg} / \mathrm{dL}$ (Ref: $0.2-1.2 \mathrm{mg} / \mathrm{dL}$ ), and alkaline phosphatase 323 U/L (Ref: 20-120 U/L). Contrast enhanced abdominal computed tomography scan demonstrated thickening and fat stranding of the gallbladder neck, concomitant intrahepatic biliary ductal dilatation, and an umbilical soft tissue deposit (image A and image B). Endoscopic retrograde cholangiopancreatography with common bile duct brushings revealed gallbladder adenocarcinoma. The patient was not a candidate for chemotherapy and died during the hospitalization from septic shock due to a bilioma and ARDS.
Sister Mary Joseph nodule is an uncommon clinical finding which may be noted on computed tomography imaging as a well-defined subcutaneous enhancing lesion. It can be either well-defined or demonstrate indefinite margins. ${ }^{1,2}$ On ultrasound, which may be used to assist in biopsy, the nodule may appear as a hypoechoic mass. ${ }^{1}$ A Sister Mary Joseph nodule should raise suspicion for metastatic or recurrent visceral malignancy and is a marker of poor prognosis. ${ }^{3}$ (doi:10.7556/ jaoa.2020.131)

\section{References}

1. Bashir O, Prince N, Ahmad S, Coveney E. Sister Joseph's nodule: imaging of a clinical sign. Appl Radiol. 2012;41(5):27-28.

2. Larentzakis A, Theodorou D, Fili K, et al. Sister Mary Joseph's nodule: three case reports. Cases J. 2008;1(1):182. doi:10.1186/ 1757-1626-1-182

3. Palaniappan M, Jose WM, Mehta A, Kumar K, Pavithran K Umbilical metastasis: a case series of four Sister Joseph nodules from four different visceral malignancies. Curr Oncol. 2010;17 (6):78-81. doi:10.3747/co.v17i6.684

(c) 2020 American Osteopathic Association
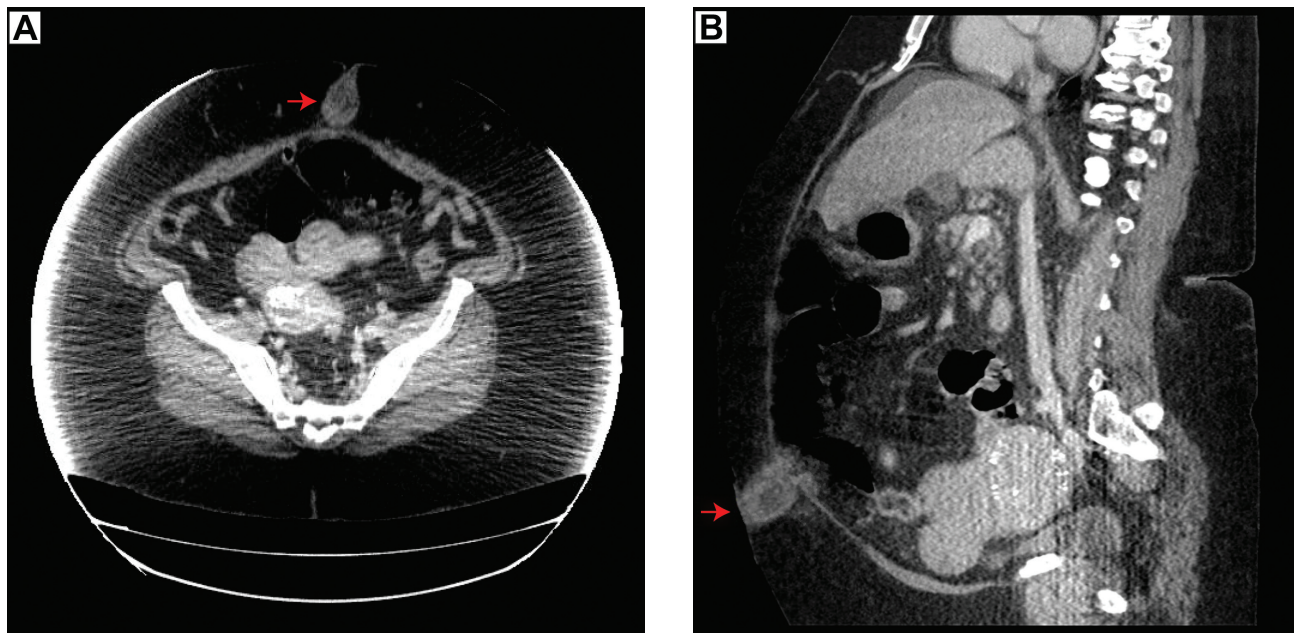